\title{
A multiple-labelling method for cells using Au nanoparticles with different shapes
}

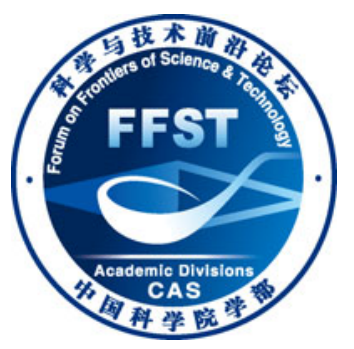

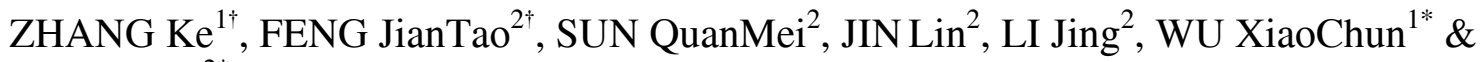 \\ HAN Dong ${ }^{2 *}$ \\ ${ }^{1}$ Chinese Academy of Sciences Key Laboratory of Standardisation and Measurement for Nanotechnology, National Centre for Nanoscience and \\ Technology, Beijing 100190, China; \\ ${ }^{2}$ Laboratory for Biological Effects of Nanomaterials and Nanosafety, National Centre for Nanoscience and Technology, Beijing 100190, China
}

Received October 23, 2012; accepted November 30, 2012; published online April 8, 2013

\begin{abstract}
An important and difficult issue is simultaneously identifying the detailed locations of various molecules on the cell surface, as this identification requires a synergistic effect between more than one molecule in a living cell. Au nanoparticles (NPs) with different shapes can be readily recognised under low vacuum scanning electron microscopy (lvSEM). Anisotropic Au nanorods (NRs) possess unique surface plasmon resonance (SPR) properties, which can be further utilised for two photon luminescence (TPL) and other optical imaging techniques. In this paper, Au NRs and Au nanooctahedra (Au NOs) are introduced as biomarkers for ICAM-1 and Integrin $\beta 1$. Combined with the advantages of lvSEM, this multiple-labelling method is a new method for studying the interactions between specific, functional molecules.
\end{abstract}

gold nanoparticles, low vacuum SEM, multiple-labelling, TPL

Citation: Zhang K, Feng J T, Sun Q M, et al. A multiple-labelling method for cells using Au nanoparticles with different shapes. Chin Sci Bull, 2013, 58: 2640-2645, doi: 10.1007/s11434-013-5794-2

The cell membrane plays a key role as a functional biointerface, physically separating cells from their microenvironment. External stimuli are perceived at the cell membrane through proteins and molecules on (or in) the cell membrane, leading to an "outside-to-inside signalling". A single signalling pathway can often modulate a broad range of cellular events, indicating that the spatiotemporal segregation of signalling molecules has a key role in activating specific downstream responses. For example, the polarised and compartmentalised nature of lymphocyte activation facilitates local signalling that leads to specific cellular responses at precise locations and times [1]. Also, the stimulus-induced leukocyte transendothelial migration, regulated by specific adhesion molecules at the sites of tissue damage, is of crucial importance for atherosclerosis [2], metastasis and tumour growth [3]. During the abovementioned pro-

$\dagger$ These authors contributed equally to this work.

*Corresponding authors (email: dhan@ nanoctr.cn; wuxc@ nanoctr.cn) cesses, recruitment of certain adhesion molecules, such as intercellular adhesion molecule-1 (ICAM-1), serve a key role, not only as adhesive substances but also as mediators of several cellular functions. Recent biochemical, proteomic, and structural studies bolster the idea that tetraspaninenriched microdomains (TEMs) at the plasma membrane account for the organisation and recruitment of protein complexes [4-8]. Francisco and co-workers [8] have demonstrated in their work that ICAM-1 is recruited for inclusion by a specially preformed TEM (CD9), which acts as endothelial adhesive platforms (EAPs) at cell-cell contact sites on the apical membrane of activated endothelial cells. EAPs are restricted to nanoscale dimensions of ca. $\sim 300 \mathrm{~nm}$ and show an increase in their density on activated cells. Additionally, several of the $\beta 1$ subfamily of integrins are largely expressed on cultured endothelial cells (ECs), which are involved in many aspects of endothelial function, including vasculogenesis, angiogenesis, wound healing, recruitment of leukocytes to sites of inflammation and regula- 
tion of thrombosis [9].

In this report, we describe a simple and feasible approach to address ICAM- 1 and Integrin $\beta 1$ simultaneously on primary culture rat cerebral microvascular endothelial cells (rCMECs) by utilising Au nanorods (NRs) and Au nanooctahedra (NOs) as markers. Low vacuum scanning electron microscopy (lvSEM) is introduced as an imaging technique that does not require any conducting coat materials, such as $\mathrm{Au}$ or $\mathrm{C}$, and thus, it can visualise the original morphology of cells in their native conformation. Furthermore, Au nanoparticles (NPs) with different shapes can be easily distinguished using lvSEM. Images with high resolution are presented. As expected, preexisting EAPs composed of TEMs and ICAM-1 in ca. $300 \mathrm{~nm}$ clusters are observed on burn serum stimulated rCMECs. Meanwhile, due to the LSPR enhancement of Au NRs, two photon luminescence (TPL) imaging [10-12], dark field scattering light imaging $[13,14]$ and SERS imaging [15] can also be obtained. Combined imaging of high resolution SEM with enhanced optical imaging will elucidate the spatial geometric distribution of molecules on cell membranes and provide further quantification of the biomolecular information.

\section{Preparation of Au NPs with conjugated antibody}

Au NRs can now be synthesised via seed mediated methods with high yield and quality. The aspect ratio (AR) of $\mathrm{Au}$ NRs can be easily tuned by changing the amount of $\mathrm{Ag}^{+}$ ions [16]. In our previous work, we successfully prepared Au NOs by introducing Au NRs as seeds [17]. Typical SEM images of $\mathrm{Au}$ NRs and Au NOs are presented in Figure 1. The length of $\mathrm{Au}$ NRs is ca. $60 \mathrm{~nm}$ with a width of ca. 15 $\mathrm{nm}$, and the edge length of Au NOs is ca. $60 \mathrm{~nm}$. The anisotropic geometry of $\mathrm{Au}$ NRs leads to the split of surface plasmon resonance (SPR) absorption bands, with one located at longer wavelength $(820 \mathrm{~nm})$, known as longitudinal SPR, and the other at a shorter wavelength $(510 \mathrm{~nm})$, known as the transverse SPR. In the case of Au NOs, only one SPR absorption appears, indicating an isotropic geometry. The original Au NRs and Au NOs are coated by a bilayer of cationic surfactants, cetyltrimethlammonium bromide (CTAB), which are positively charged [18]. Next, the positively charged surface of the NPs is changed to a negatively charged surface by exposing the NPs to poly(sodium $p$-styrensulphonate) (PSS, $\left.M_{\mathrm{W}} 70000\right)$ polyelectrolyte solutions [19]. Subsequently, the nanoparticles are dispersed in $N$-(2-hydroxyethyl)piperazine- $N$ '-2-ethanesulphonic acid (HEPES) solution ( $\mathrm{pH}$ 7.4) for bonding with an antibody. The PSS coated NPs are finally mixed with antibody solutions, which are diluted in the same HEPES buffer, and allowed to react overnight. The antibodies are most likely bound to the PSS coated nanoparticles by electrostatic interactions $[13,20]$ and form antibody conjugated Au NPs.

In this study, Au NRs and Au NOs are typically chosen as markers among different morphological Au NPs. Theoretically, these markers are entirely interchangeable for binding antibodies, except Au NPs with a particular shape and unique properties can be intentionally used for specific proteins (or molecules) with predictable distributions. In this study, Au NRs are designated biomarkers for ICAM-1 because of the significant change observed after stimulation. By introducing the LSPR enhancement, optical images of $\mathrm{Au}$ NRs are possible.

\section{Procedure and SEM characterisations of rCMECs labelled by different Au NPs}

The procedure for immune labelling rCMECs utilising antibodies conjugated with Au NPs is illustrated in Figure 2. The cultured rCMECs (The animals' care was in accordance with institutional guidelines. The protocol used in this manuscript was agreed to in advance by the Ethics Committees of National Centre for Nanoscience and Technology, China) are first prefixed with $0.5 \%$ glutaraldehyde for $2 \mathrm{~h}$ and then washed within $150 \mathrm{mmol} / \mathrm{L}$ glycine, to bond the residual
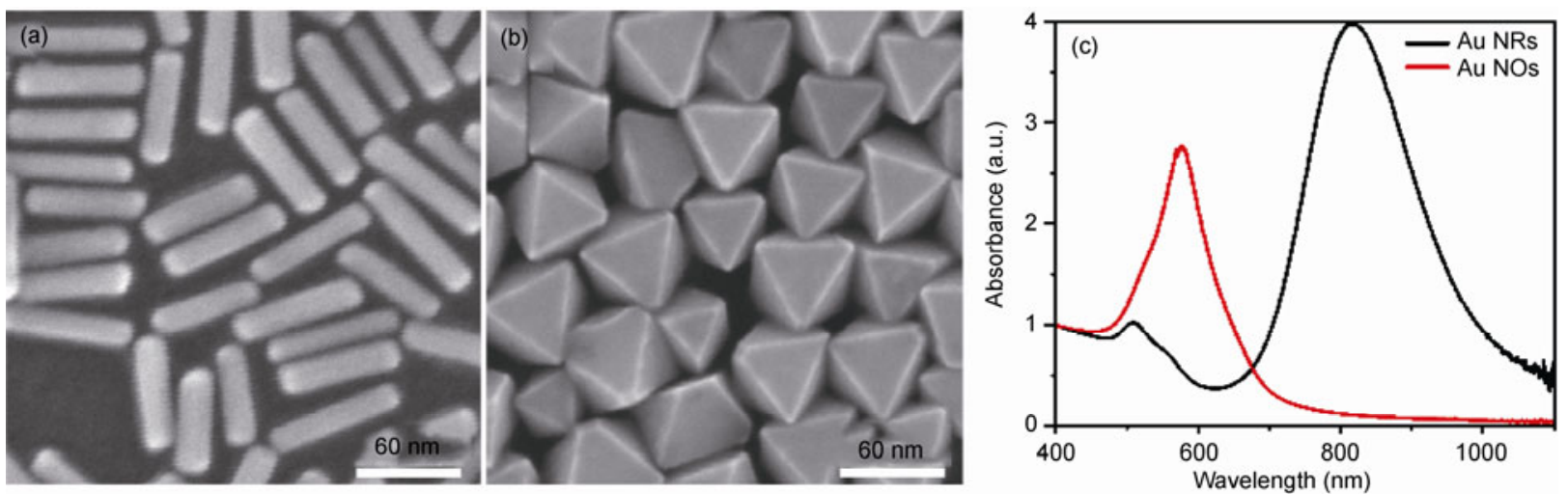

Figure 1 SEM images of prepared Au NRs (a), Au NOs (b) and the corresponding UV-vis-NIR absorption spectra in water (c). The spectra are normalised at $400 \mathrm{~nm}$. 


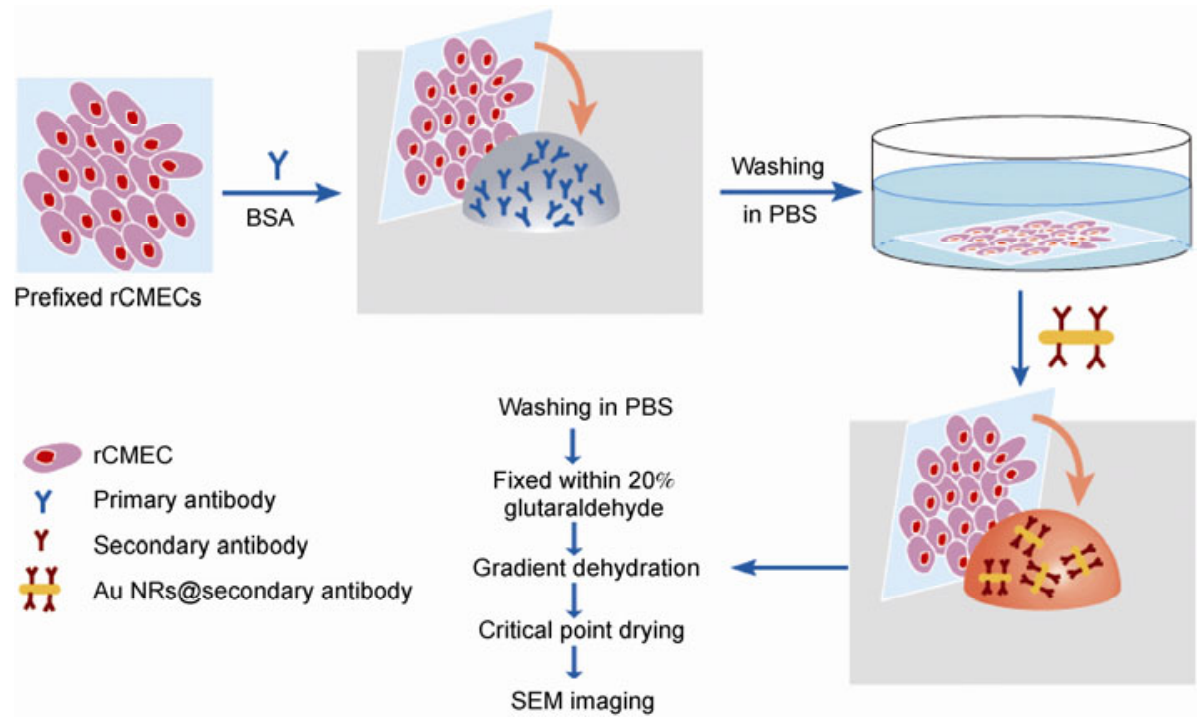

Figure 2 Schematic pictures of the labelling process for ICAM-1 on rCMECs membrane surfaces by Au NRs modified with a Goat anti-Mouse antibody.

aldehyde. Second, the traditional immunofluorescent method is used for cross-linking the proteins on the cell surfaces by primary antibody and blocking of the nonspecific adsorption sites by $0.01 \mathrm{~g} / \mathrm{mL}$ BSA for $4 \mathrm{~h}$ at $37^{\circ} \mathrm{C}$. The rCMECs are washed in PBS (pH 7.0) buffer, to remove the unbound antibodies, followed by incubation with a secondary antibody conjugated $\mathrm{Au}$ NPs for $4 \mathrm{~h}$ at $37^{\circ} \mathrm{C}$. Next, the samples were washed with PBS to remove the unbound and nonspecifically adsorbed Au NPs. Finally, the samples were fixed with glutaraldehyde, dehydrated in graded ethanol solutions, dried by critical point drying, and imaged by lvSEM.

To verify the feasibility of this approach, Au NRs and Au NOs are chosen to map the relationship between ICAM-1 and Integrin $\beta 1$, both in normal and pathological states. As shown in Figure 3(a), obvious tight junctions between neighbouring rCMECs exist and are the basis of the blood brain barrier (BBB). The apical surfaces of rCMECs are smooth and neat, with typical microvilli and a cell morphology that is still plump, as observed in culture medium after CPD. Both the Au NRs and Au NOs labelling ICAM-1 and Integrin $\beta 1$ can be distinguished (Figure 3(c), (e), (g)) and exhibit significant variation compared to the corresponding control experiment that shows nonspecific adhesion of Au NRs and Au NOs (data not shown).

To obtain greater insight into the cellular distribution of ICAM- 1 and Integrin $\beta 1$, cell-cell contact sites are magnified in Figure 3(d), (f) and (h) and correspond to the red squares in Figure 3(c), (e) and (g). As the lvSEM images demonstrated, ICAM-1 is constitutively expressed at low levels on normal endothelial cells, and Integrin $\beta 1$ is largely expressed and enriched at cell-cell contact sites [21-23]. After coculturing the rCMECs with $2 \%$ burn serum overnight, for an inflammatory stimulation, the morphology of the cells changes greatly. Many vesicles aggregate just un- derneath the cell membrane (Figure 4). ICAM-1 molecules on the cell surface increases significantly and aggregate together on the apical side of the cell membrane at the cell-cell contact area, forming nanoclusters measuring ca. $\sim 400 \mathrm{~nm}$ in diameter (Figure 4(c) and (d)).

Previous studies have shown that ICAM-1 molecules are clustered into membrane rafts and form actin-based structures at the contact area upon leukocytes binding to prevent detachment under hemodynamic flow [24-27]. Recently, Sánchez-Madrid and co-workers [25] demonstrated that the recruitment of ICAM-1 molecules occurs before leukocyte binding, and the apical plasma membrane of living ECs possesses specialised microdomains containing tetraspanins (CD151) and ICAM-1 molecules, which are termed endothelial adhesive platforms (EAPs). This particular type of TEM promotes ICAM-1 molecules aggregating at the nanoscale level, thus forming nanoclusters to enhance their adhesive properties during leukocyte adhesion to endothelium [8], which is consistent with our results (Figure 4(d) arrow headed). Several tetraspanins have been proven to associate with integrins of the $\beta 1$ subfamily [28-31] and subsequently modulate endothelial cell motility by forming complexes. As Ashman and co-workers [32] have shown, integrin plays a key role in cell migration and modulates angiogenesis but has no detectable effect on neutrophil transendothelial migration when anti CD151 mAb is introduced. It can be inferred that the expression of integrins on the endothelial cell membrane may not be remarkably affected by inflammatory simulation by $2 \%$ burn serum. And the level of Integrin $\beta 1$ is not up- or down-regulated significantly by comparing the SEM images with or without stimulating (Figure 4(f)). CD151 is a well-known member of the tetraspanins, and associates with both ICAM-1 and Integrin $\beta 1$ to form complexes and further modulate the cell functions [33]. Thus, the localisation of ICAM-1 and Integrin 

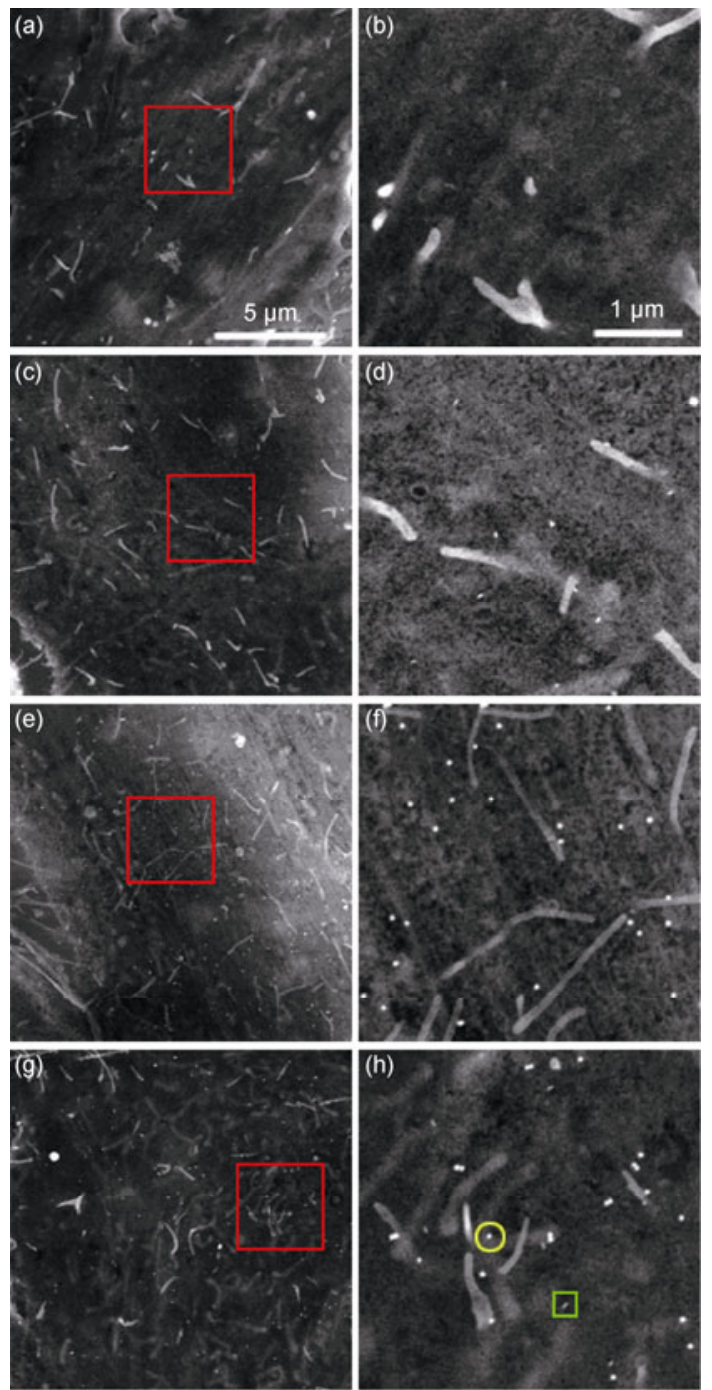

Figure 3 LvSEM images of rCMECs. (a) Nude rCMECs apical surface, (c) labelled by goat anti-mouse IgG conjugated Au NRs for ICAM-1, (e) labelled by goat anti-rabbit IgG conjugated Au NOs for Integrin $\beta 1$, (g) double labelled by goat anti-mouse IgG conjugated Au NRs and goat anti-rabbit IgG conjugated Au NOs. (b), (d), (f), and (h) are zoomed in images of the corresponding red squares in (a), (c), (e) and (g). The green rectangle and yellow circle in (h) indicate Au NR and Au NO under SEM, respectively.

$\beta 1$ could partially overlap when co-labelled (Figures 3(h) and $4(h))$.

\section{TPL images of rCMECs labelled by Au NRs}

Because Au NRs show enhanced LSPR emissions, TPL images could be taken for these identical cell samples. Mode-locked Ti:sapphire femtosecond laser pulse (Tsunami, Spectra-Physics) at $785 \mathrm{~nm}$ is introduced as an excitation source. The pulse width is approximately $120 \mathrm{fs}$ with a repetition frequency of $82 \mathrm{MHz}$. Emitted light is collected in the range between 400 and $700 \mathrm{~nm}$. Figure 5(c) shows strong TPL images for burn serum stimulated rCMECs
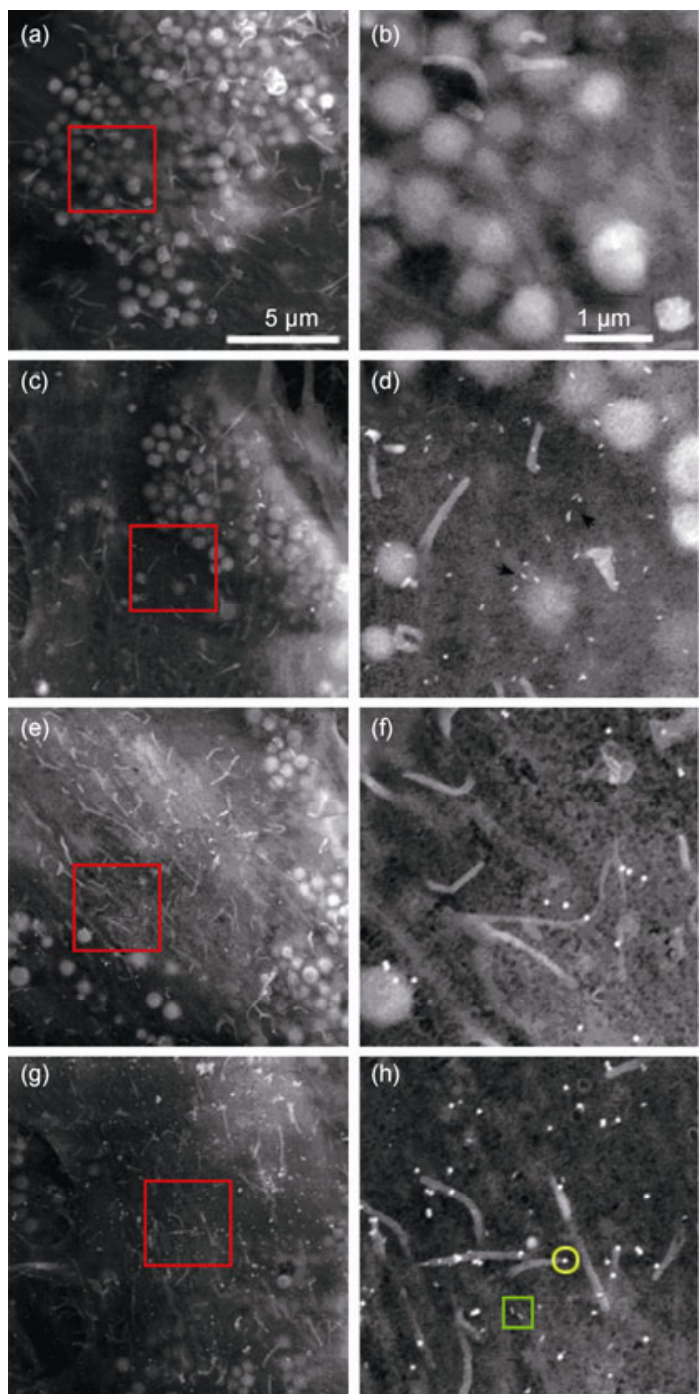

Figure 4 LvSEM images of rCMECs stimulated by $2 \%$ burn serum overnight. (a) Nude stimulated rCMECs apical surface, (c) labelled by goat anti-mouse IgG conjugated Au NRs for ICAM-1, (e) labelled by goat anti-rabbit IgG conjugated Au NOs for Integrin $\beta 1,(\mathrm{~g})$ double labelled by goat anti-mouse $\operatorname{IgG}$ conjugated $\mathrm{Au}$ NRs and goat anti-rabbit IgG conjugated Au NOs. (b), (d), (f), and (h) are zoom-in images of the corresponding red squares in (a), (c), (e) and (g). The green rectangle and yellow circle in (h) stand for Au NRs and Au NO under SEM, respectively.

labelled by an Au NRs with antibody conjugate, whereas control experiments show an almost black background (Figure 5(a)) and a negligible interference signal from strong TPL images for burn serum stimulated rCMECs labelled by an Au NRs with antibody conjugate, whereas control experiments show an almost black background nonspecifically adsorbed Au NRs (Figure 5(b)). The pathological rCMECs labelled by Au NOs@antibody conjugate for Integrin $\beta 1$ also exhibit negligible TPL signals (Figure $5(d)$ ), which indicates the TPL signals solely originate from the SPR enhancement of AuNRs. The TPL results coincide with the lvSEM and traditional immunofluorescence. TPL images show protein distribution information in microscale. And when combined with the lvSEM images in nanoscale 


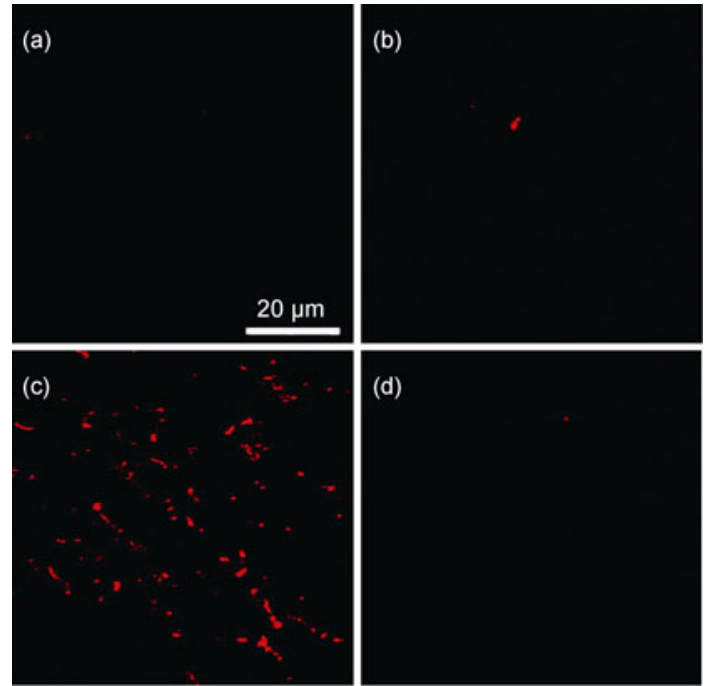

Figure 5 TPL images of (a) nude stimulated rCMECs, (b) stimulated rCMECs labelled by Au NRs via nonspecific adsorptions, (c) stimulated rCMECs labelled by Au NRs for ICAM-1, and (d) stimulated rCMECs labelled by Au NOs for Integrin $\beta 1$.

from the same sample, these two images complement and verify each other to give insightful bioinformation.

We conducted traditional immunofluorescence for comparison (Figure 6). The results are consistent with the $\mathrm{Au}$ NPs labelling method we developed above. After burn serum stimulation, the expression of ICAM-1 greatly increases and forms a sharp contrast with the control, while Integrin $\beta 1$ expression shows moderate up-regulation. The fluorescent approach is somewhat qualitative, and other characterisations are needed to establish a relationship between these two proteins.

\section{Conclusions}

In this work, we provide a simple and feasible approach to
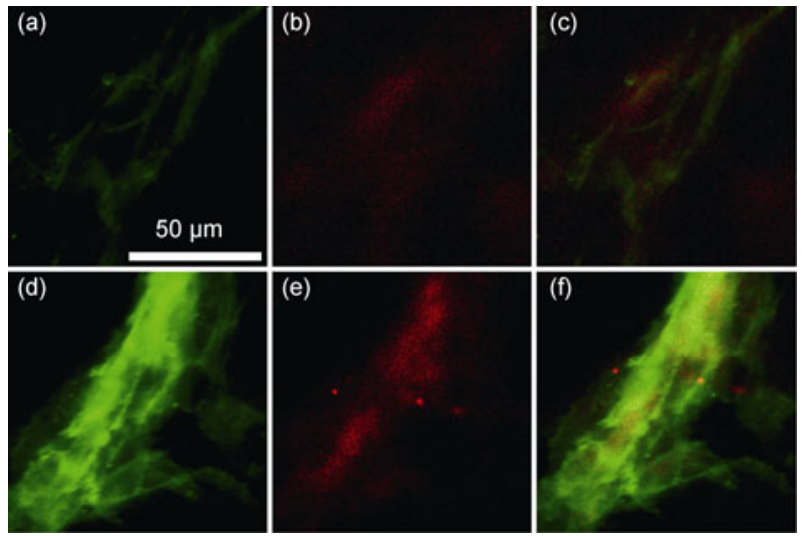

Figure 6 Fluorescence images of ICAM-1 (FITC) and Integrin $\beta 1$ (TRITC) expressed in normal rCMECs ((a)-(c)) and stimulated rCMECs ((d)-(f)). (c) and (f) are the combined images of (a), (b) and (d), (e), respectively. The scale bar applies for all the images. label two or more proteins simultaneously on the cell membrane using Au NPs of different shapes. LvSEM technology ensures that the high resolution images of the cell in its original condition could be achieved without prespinning conductive material, such as Au or C. Also, by virtue of the SPR property of Au NRs, TPL images could be obtained for identical samples, which enables us to obtain protein localisation in micro/nano scales and better understand the cell's functions and activities. Due to the SPR enhancement of Au NRs, SERS spectra may be acquired, and we can acquire more subtle information about the local micro-environment of the cell. It may also be useful to further quantify aspects of cell biology and provide the possibility for in situ imaging using SEM and optical techniques.

This work was supported by the National Basic Research Program of China (2012CB933800 and 2011CB932802), and the National Natural Science Foundation of China (21173056 and 21201041).

1 Viola A, Gupta N. Tether and trap: Regulation of membrane-raft dynamics by actin-binding proteins. Nat Rev Immunol, 2007, 7: 889-896

2 Buul J D, Kanters E, Hordijk P L. Endothelial signaling by Ig-like cell adhesion molecules. Arterioscler Thromb Vasc Biol, 2007, 27: 1870-1876

3 Yasuda M, Tanaka Y, Tamura M, et al. Stimulation of $\beta 1$ integrin down-regulates ICAM-1 expression and ICAM-1-dependent adhesion of lung cancer cells through focal adhesion kinase. Cancer Res, 2001, 61: 2022-2030

4 Hemler M E. Tetraspanin proteins mediate cellular penetration, invasion, and fusion events and define a novel type of membrane microdomain. Annu Rev Cell Dev Biol, 2003, 19: 397-422

5 Hemler M E. Tetraspanin functions and associated microdomains. Nat Rev Mol Cell Biol, 2005, 6: 801-811

6 Naour F L, André M, Boucheix C, et al. Membrane microdomains and proteomics: Lessons from tetraspanin microdomains and comparison with lipid rafts. Proteomics, 2006, 6: 6447-6454

7 Min G W, Wang H B, Sun T T, et al. Structural basis for tetraspanin functions as revealed by the cryo-EM structure of uroplakin complexes at 6-Å resolution. J Cell Biol, 2006, 173: 975-983

8 Barreiro O, Zamai M, Yáñez-Mó M, et al. Endothelial adhesion receptors are recruited to adherent leukocytes by inclusion in preformed tetraspanin nanoplatforms. J Cell Biol, 2008, 183: 527-542

9 Shattil S J, Ginsberg M H. Perspectives series: Cell adhesion in vascular biology. Integrin signaling in vascular biology. J Clin Invest, 1997, 100: 1-5

10 Durr N J, Larson T, Smith D K, et al. Two-photon luminescence imaging of cancer cells using molecularly targeted gold nanorods. Nano Lett, 2007, 7: 941-945

11 Wang $\mathrm{H} \mathrm{F}$, Huff $\mathrm{T} \mathrm{B}$, Zweifel $\mathrm{D}$ A, et al. In vitro and in vivo two-photon luminescence imaging of single gold nanorods. Proc Natl Acad Sci USA, 2005, 102: 15752-15756

12 Tong L, He W, Zhang Y S, et al. Visualizing systemic clearance and cellular level biodistribution of gold nanorods by intrinsic two-photon luminescence. Langmuir, 2009, 25: 12454-12459

13 Huang X, El-Sayed I H, Qin W, et al. Cancer cell imaging and photothermal therapy in the near-infrared region by using gold nanorods. J Am Chem Soc, 2006, 128: 2115-2120

14 Chanda N, Shukla R, Katti K V, et al. Gastrin releasing protein receptor specific gold nanorods: Breast and prostate tumor avid nanovectors for molecular imaging. Nano Lett, 2009, 9: 1798-1805

15 Park H, Lee S, Chen L, et al. SERS imaging of HER2-over expressed MCF7 cells using antibody-conjugated gold nanorods. Phys Chem Chem Phys, 2009, 11: 7444-7449 
16 Guo L F, Murphy C J. Fine-tuning the shape of gold nanorods. Chem Mater, 2005, 17: 3668-3672

17 Xiang Y J, Wu X C, Liu D F, et al. Tuning the morphology of gold nanocrystals by switching the growth of $\{110\}$ facets from restriction to preference. J Phys Chem C, 2008, 112: 3203-3208

18 Nikoobakht B, El-Sayed M A. Evidence for bilayer assembly of cationic surfactants on the surface of gold nanorods. Langmuir, 2001, 17: 6368-6374

19 Gittins D I, Caruso F. Tailoring the polyelectrolyte coating of metal nanoparticles. J Phys Chem B, 2001, 105: 6846-6852

20 Wang C G, Chen Y, Wang T T, et al. Biorecognition-driven selfassembly of gold nanorods: A rapid and sensitive approach toward antibody sensing. Chem Mater, 2007, 19: 5809-5811

21 Balsamo J, Ernst H, Zanin M K, et al. The interaction of the retina cell surface $N$-acetylgalactosaminylphosphotransferase with an endogenous proteoglycan ligand results in inhibition of cadherinmediated adhesion. J Cell Biol, 1995, 129: 1391-1401

22 Peñas P F, García-Díez A, Sánchez-Madrid F, et al. Tetraspanins are localized at motility-related structures and involved in normal human keratinocyte wound healing migration. J Invest Derm, 2000, 114: 1126-1135

23 Mittelbrunn M, Yáñez-Mó M, Sánchez-Madrid F. Tetraspanins and intercellular interactions. Microcirculation, 2001, 8: 153-168

24 Tilghman R W, Hoover R L. E-selectin and ICAM-1 are incorporated into detergent-insoluble membrane domains following clustering in endothelial cells. FEBS Lett, 2002, 525: 83-87

25 Barreiro O, Yáñez-Mó M, Serrador J M, et al. Dynamic interaction of VCAM-1 and ICAM-1 with moesin and ezrin in a novel endothelial docking structure for adherent leukocytes. J Cell Biol, 2002, 157: 1233-1245
26 Barreiro O, Yáñez-Mó M, Sala-Valdés M, et al. Endothelial tetraspanin microdomains regulate leukocyte firm adhesion during extravasation. Blood, 2005, 105: 2852-2861

27 Buul J D, Allingham M J, Samson T, et al. RhoG regulates endothelial apical cup assembly downstream from ICAM1 engagement and is involved in leukocyte trans-endothelial migration. J Cell Biol, 2007, 178: 1279-1293

28 Berditchevski F, Zutter M M, Hemler M E. Characterization of novel complexes on the cell surface between integrins and proteins with 4 transmembrane domains (TM4 proteins). Mol Biol Cell, 1996, 7: 193-207

29 Nakamura K, Iwamoto R, Mekada E. Membrane-anchored heparinbinding EGF-like growth factor (HB-EGF) and diphtheria toxin receptor-associated protein (DRAP27)/CD9 form a complex with integrin $\alpha 3 \beta 1$ at cell-cell contact sites. J Cell Biol, 1995, 129: 16911705

30 Porter J C, Hogg N. Integrins take partners: Cross-talk between integrins and other membrane receptors. Trends Cell Biol, 1998, 8: 390-396

31 Klein-Soyer C, Azorsa D O, Cazenave J P, et al. CD9 Participates in endothelial cell migration during in vitro wound repair. Annu Rev Cell Dev Biol, 2000, 20: 360-369

32 Sincock P M, Fitter S, Parton R G, et al. PETA-3/CD151, a member of the transmembrane 4 superfamily, is localised to the plasma membrane and endocytic system of endothelial cells, associates with multiple integrins and modulates cell function. J Cell Sci, 1999, 112: 833-844

33 Lammerding J, Kazarov A R, Huang H, et al. Tetraspanin CD151 regulates $\alpha 6 \beta 1$ integrin adhesion strengthening. Proc Natl Acad Sci USA, 2003, 100: 7616-7621

Open Access This article is distributed under the terms of the Creative Commons Attribution License which permits any use, distribution, and reproduction in any medium, provided the original author(s) and source are credited. 